\title{
Strain-Dependent Dynamic Mechanical Properties of Kevlar to Failure: Structural Correlations and Comparisons to other Polymers
}

\author{
Shilpa N. Raja ${ }^{1,2,}$ Sandip Basu ${ }^{3}$, Aditya M. Limaye ${ }^{4}$, Christina M. Hyland ${ }^{4}$, Turner J. \\ Anderson ${ }^{5}$, Liwei Lin ${ }^{5}$, A. Paul Alivisatos ${ }^{1,2,6}$, Robert O. Ritchie $*^{1,2,5}$ \\ ${ }^{1}$ Materials Sciences Division, Lawrence Berkeley National Laboratory, Berkeley, CA 94720 \\ ${ }^{2}$ Department of Materials Science \& Engineering, University of California, Berkeley, CA 94720 \\ ${ }^{3}$ Agilent Technologies, 4330 W. Chandler Blvd, Chandler, AZ 85226 \\ ${ }^{4}$ Department of Chemical Engineering, University of California, Berkeley, CA 94720 \\ ${ }^{5}$ Department of Mechanical Engineering, University of California, Berkeley, CA 94720 \\ ${ }^{\square}$ Department of Chemistry, University of California, Berkeley, CA 94720 \\ *Corresponding author email: roritchie@lbl.gov
}

\begin{abstract}
The processing of Kevlar to certain strengths by hot-drawing can benefit by quantitative understanding of the correlation between structural and mechanical properties during the predrawing process. Here, we use a novel continuous dynamic analysis (CDA) to monitor the evolution in storage modulus and loss factor of Kevlar 49 fibers as a function of strain via a quasi-static tensile test. Unlike traditional dynamic mechanical analysis, CDA allows the tracking of strain-dependent mechanical properties until failure. The obtained dynamic viscoelastic properties of Kevlar 49 are correlated with structural data obtained from synchrotron radiation analysis and with Raman scattering frequencies. Rate-dependent stress-strain results from Kevlar are compared to Nomex, spider silk, polyester and rubber, and provide insight into how the mechanical properties of Kevlar originate from its characteristic structural features. We find that as the storage modulus of Kevlar is essentially equal to the Young's modulus, the measured quantitative relationships between storage modulus and strain can provide insights into the tuning of the mechanical properties of aramid materials for specific applications.
\end{abstract}


Keywords: Kevlar; Aramid; Polymer; Dynamic Mechanical Analysis; Structure Property Relationship

\section{Introduction}

Poly(p-phenylene terephthalamide), commonly called Kevlar, is a highly crystalline polyaramid fiber with exceptionally high strength and remarkable thermal stability, widely used in stress-bearing applications such as bulletproof body armor, shielding for sports equipment, and fiber-reinforced polymer composites in the aerospace industry (1). Kevlar is sold in several different grades, each of which is optimized by hot-drawing to develop specific properties (1). For example, Kevlar 129 has the highest strength, while Kevlar 119 is more ductile than the other grades (1). In this study, we focus on Kevlar 49, a higher-modulus grade of Kevlar designed for load-bearing applications in cables, cloth, and aerospace components (1). While many studies of the quasi-static behavior of the various types of Kevlar fiber are available (2), there have been far fewer investigations on their dynamic mechanical properties (3). Those that do exist characterize the storage and loss modulus as a function of temperature without considering the effects of continuously increasing strains, mainly due to the limitations of current dynamic mechanical analysis (DMA) (3). DMA tests can only be carried out at low harmonic displacements to ensure linear viscoelasticity, severely limiting the ability to characterize the viscoelastic response of materials past their yield point (3).

On the other hand, the technique of continuous dynamic analysis (CDA) combines the advantages of dynamic mechanical analysis with those of the quasi-static tensile test to quantify the evolution of viscoelastic properties as a continuous function of strain. This is achieved through application of a small harmonic $(20 \mathrm{~Hz})$ strain during the tensile deformation. Because 
the harmonic strain is much smaller in magnitude than the applied quasi-static strain, it remains within the limits of linear viscoelasticity and does not affect the behavior of a quasi-static tensile test. Here, using a specialized electromechanical load cell capable of applying small but continually increasing periodic forces, we employ CDA to monitor the dynamic properties of Kevlar 49 fibers as a function of their strain to failure. We then quantitatively correlate our results to the structural tensile data of Kevlar from previous studies utilizing wide-angle $\mathrm{x}$-ray diffraction (WAXD) (5) and Raman spectroscopy (6), to provide insight into the molecular mechanisms by which Kevlar tolerates stress. The dynamic mechanical behavior of Kevlar is further compared to those of other key structural polymers to quantitatively discern its dynamic responses against known benchmarks. As such, this study presents quantitative information on how the drawing of Kevlar changes its mechanical properties, thereby providing guidelines for the systematic optimization of this important engineering fiber.

\section{Continuous Dynamic Analysis of Kevlar and Nomex}

Kevlar is a para-aramid polymer which has superior mechanical properties to Nomex, a flame-resistant meta-aramid polymer. The extensive hydrogen bonds arising from Kevlar's parasubstituted backbone link adjacent chains to form sheets. These stacked sheets roll into "microfibrils," which bundle together to form the bulk Kevlar fiber. This hierarchical structure enables Kevlar to have more aligned crystalline structure than most structural polymers (1). Nomex, on the other hand, is a constitutional isomer of Kevlar. Its amine functional group is situated with the meta orientation on the phenyl ring in a monomer unit, as opposed to the para configuration in Kevlar. This small difference in molecular structure changes the optimal bond angles of phenyl-nitrogen and phenyl-carbon bonds in Nomex, resulting in a "crumpled" chain structure that cannot crystallize into stacked sheets (7). This causes it to take on a "jungle gym" 
structure (7), with randomly stacked polymer chains, and a resulting lower overall crystallinity than Kevlar (7).

Here we consider how the loss factor varies between these polymers with differing monomer structure. The loss factor is the ratio of the loss modulus to the storage modulus, and can be thought of as a ratio relating the viscously dissipated energy in a material to the elastically stored energy. At the relatively low frequencies of perturbation applied here, the dissipative contribution to the mechanical response of the polymer arises as polymer chains align along the axis of applied tension. For polymers in general, the loss factor as a function of the strain can be expected to increase up until the critical strain at which the chains become effectively aligned, whereupon a reduction in loss factor occurs. The absolute value of the peak in the strain curve, and the value of the strain at which it occurs, will vary depending upon both the molecular structure and the degree of order of the polymer. In amorphous polymers, entangled chains can slip past each other under modest tensile stress, dissipating energy through viscous friction. Highly crystalline polymers, on the other hand, generally begin with their crystalline units well aligned with the tensile axis (8).

The measured loss factor and stress-strain curve to failure of Kevlar 49, shown in Fig. 1A, exhibits mostly linear behavior with small nonlinearities to failure with a Young's modulus of $168 \pm 4 \mathrm{GPa}$. Initially, the loss factor begins to increase due to chain unraveling, reaching a maximum at a strain of $0.5 \%$, marked as deformation regime I on the curve. As the microfibrils in Kevlar become more aligned in response to increased strain, the loss factor begins to decrease, beginning in regime II. Throughout this regime, the fiber orientation is stabilized (i.e., the fiber is increasingly oriented at a microstructural level), and each of the microfibrils in a Kevlar fiber (1) act similarly to a simple harmonic spring, storing applied stress as elastic energy. A slight 
flattening-out of the loss factor curve after about $\sim 1.5 \%$ strain occurs due to saturation of microfibril alignment.

The corresponding measured loss factor and stress-strain curves for Nomex are shown in Figure 1B. The Young's modulus in the elastic region, Regime I, was measured to be $11.5 \pm 1.4$ $\mathrm{GPa}$, an order of magnitude lower than that of Kevlar. In this regime, as above, chains begin to unravel, increasing structural disorder. Regime II begins after the loss factor reaches a maximum, and the "jungle gym" (7) structure in Nomex begins to align with the tensile axis. As above, a very slight leveling-off of the loss factor decrease is observed with increasing strain in this regime, due to molecular-level chain alignment reaching a maximum. The "jungle gym" structure limits the achievable crystallinity; as such the stress-strain curve of Nomex is very different from that of Kevlar, exhibiting nearly $150 \%$ higher ductility and a large plastic region before failure.

Repeat tests with more than five fibers of each of Kevlar and Nomex showed less than $10 \%$ variation in all parameters (see Figs. 1-2 in Supplementary Information).

\section{CDA of Polyester, Dragline Silk, and Rubber and Comparison to Kevlar}

Figure 2A shows stress-strain curves of Kevlar, Nomex, polyethylene terephthalate (PET), Argiope spider silk, and Kraton SEBS at strains up to failure. Spider silk, a semi-crystalline natural material composed of protein fibroins, is known to have specific tensile strength higher than that of many steels (9). Polyethylene terephthalate (PET) is a typical semi-crystalline polymer consisting of spherulites, or crystalline lamellae linked by amorphous tie-chains (10). Finally, poly(styrene- $b$-ethylene-butylene- $b$-styrene) (SEBS), a tri-block copolymer commonly 
used in shoe soles, asphalt, and adhesives (11), adapts a lamellar structure of alternating polystyrene (PS) and poly(ethylene-butylene) (PEB) domains (12).

Due to the strong hydrogen-bonded polymer chain network found in Nomex and Kevlar, these polymers are more crystalline than spider silk and PET and hence have higher yield stresses, as seen in Fig. 2A. The Young's moduli for spider silk, PET, and SEBS were measured as $26.5 \pm 3.4 \mathrm{GPa}, 10.5 \pm 0.4 \mathrm{GPa}$, and $16.3 \pm 12.9 \mathrm{MPa}$, respectively, i.e., between 0.5-4 orders of magnitude lower than Kevlar $49(168 \pm 4 \mathrm{GPa})$. As the storage modulus quantifies the effectiveness of elastic energy storage, high storage moduli are generally found in crystalline polymers such as Kevlar as highly oriented polymer chains act like harmonic springs rather than dashpots, which dissipate stress through viscous friction (9). Conversely, amorphous polymers have low storage moduli, since much of the energy supplied to them during tensile deformation is dissipated during chain slippage and entanglement.

Figure $2 \mathrm{~B}$ shows the storage modulus as a function of strain in all of the polymers examined in this work. As expected, due to increasing polymer chain alignment during a tensile test, the storage modulus in all cases increases with strain, reaching levels of 200-350\% above its original value just before failure. Kevlar, due to its extremely high degree of crystallinity, has a maximum storage modulus of $268.5 \mathrm{GPa}$, more than two orders of magnitude greater than that of SEBS, 0.32 GPa. A semi-crystalline polymer like Nomex or PET, on the other hand, reaches a maximum storage modulus of $\sim 40 \mathrm{GPa}$. It can be concluded that the oriented, crystalline chains in Kevlar store energy much more effectively than the completely amorphous Kraton SEBS or crumpled chains in Nomex. All of the polymers except Kevlar exhibit a minimum in the storage modulus near the onset of deformation most likely due to chain unraveling or mesoscale processes that decrease chain alignment, including lamellar kinking in SEBS (13), unfolding of 6 
small lamellae in PET, or unraveling of $\beta$-sheets in spider silk (10). This minimum is not present in Kevlar due to its much higher relative degree of crystallinity which prevents the domination of unfolding processes.

The storage modulus curve in Kevlar is approximately linear, increasing with a slope of 4530 GPa per unit strain. Because the loss modulus of Kevlar is four orders of magnitude lower than the storage modulus, the storage modulus can, to a high degree of precision, be taken as the Young's modulus in this material. Therefore, we have essentially quantitatively characterized the change in Young's modulus with strain in Kevlar. This information is important to designers of structural aramids for practical applications as it has not always been clear how to tune the Young's modulus of Kevlar. We believe that the use of the continuous dynamic analysis (CDA) applied here provides insight into the mechanical behavior of these polymeric fibers which can find application in the future design of materials that use Kevlar as a filler or as a majority component.

Figure $2 \mathrm{C}$ shows the loss factor curves for all polymers examined in this study, which all share a common shape, increasing to a maximum near the yield point and then decreasing continuously until sample failure. Based on this general shape, both the loss factor maximum and the strain at which the maximum is reached provide quantitative data about the structure of the polymer in question. For example, Kevlar has the lowest maximum loss factor, peaking at 0.038 , and reaches this peak at a strain of $0.5 \%$ as its stacked sheet structure begins to be fully aligned, thereby dissipating a minimal amount of energy due to chain unraveling and entanglement. In contrast, the maximum loss factor in SEBS is 0.3 , reaching at a strain of $15 \%$ as the amorphous structure of SEBS spends a much longer time aligning with the tensile axis, dissipating energy viscously along the way. A semi-crystalline polymer such as PET lies between these two 
extremes, with a maximum loss factor of 0.1 at a strain of approximately $2 \%$. This maximum is due to the slippage of amorphous tie chains in the polymer while the crystalline domains orient themselves with the tensile axis with less viscous dissipation of energy than that of a completely amorphous polymer. For similar reasons, comparable values are seen for Nomex and dragline spider silk.

\section{Quantitative Correlations of Dynamic Properties with Structural Changes in Kevlar}

Figure 3 quantitatively correlates CDA data on the storage modulus and the loss factor of Kevlar with results on other polymers, as documented in the in situ SAXS/WAXD structural data in ref. (5). Curve 1 (blue) correlates the Herman's orientation factor (a commonly used measure of polymer chain orientation) of the (200) crystal reflection in Kevlar. The values of storage modulus observed in this study yield an increase of $786 \mathrm{GPa}$ per unit change in orientation. As expected, the storage modulus increases approximately linearly with orientation, because more oriented chains have more covalent bonds aligned with the tensile axis. Analogously, the Curve 2 (red) correlates the decrease in loss factor with a greater degree of molecular orientation, yielding a drop of 0.4 loss factor units per unit increase in orientation, as defined by the Herman orientation factor (5). This is due to the fact that more oriented fibers possess fewer modes for viscous dissipation (slippage, chain entanglement, and others) than their more amorphous counterparts.

Curve 3 (green) in Fig. 3 correlates an increased storage modulus with a lower Raman absorption frequency of the $\mathrm{C}=\mathrm{C}$ bond in Kevlar, corresponding to a greater magnitude of bond stretching (5). Data for Kevlar was compared with Raman measurements on other polymers, as

documented in ref (6). The storage modulus for Kevlar increases by $11 \mathrm{GPa} / \mathrm{cm}^{-1}$ shift in the 
Raman band, since the covalent bonds, which act similar to harmonic springs, are stretched more with increasing fiber alignment. The error bars for all three curves reflect uncertainties due to noise in the CDA data, and are larger for the loss factor data due to the sharp maximum reached in Kevlar at a strain of $0.5 \%$. These structural correlations show quantitatively how the stiffness of Kevlar increases and the elasticity of its mechanical response increases as the fibrillar microstructure becomes more oriented along the tensile axis.

It should be noted that though these assessments were performed on the same fiber material, they represent comparisons of our Kevlar data with literature structural data taken on other samples. Respective strain rates were not always the same although they only differed by less than an order of magnitude, specifically a $~ 50-70 \%$ difference, for both Raman (5) and WAXD (6) measurements, which we believe does not alter the conclusions; likewise, we believe that any differences in sample size also do not significantly affect our conclusions.

In summary, using continuous dynamic analysis (CDA), which overcomes the limitations of traditional DMA by using small harmonic forces applied throughout a tensile test, we have provided a quantitative characterization of the dynamic mechanical properties of Kevlar 49 fibers as a function of continuously increasing strain until failure. This information provides key insight on the fiber strains over which certain molecular and microstructural mechanisms of deformation are active in this structural aramid. Using data from previous studies $(5,6)$ on structural changes from synchrotron and Raman studies, structural correlations and comparisons are also made with other polymers of different structure to provide further insight on the relationship between microstructure and deformation in these fibers. Since the storage modulus is essentially equal to the Young's modulus for Kevlar due to its highly crystalline structure, the roughly quantitative relationship between the storage modulus and strain presented here can 
provide key insights into the engineering of "tuned" aramid materials with properties tailored for specific applications. 

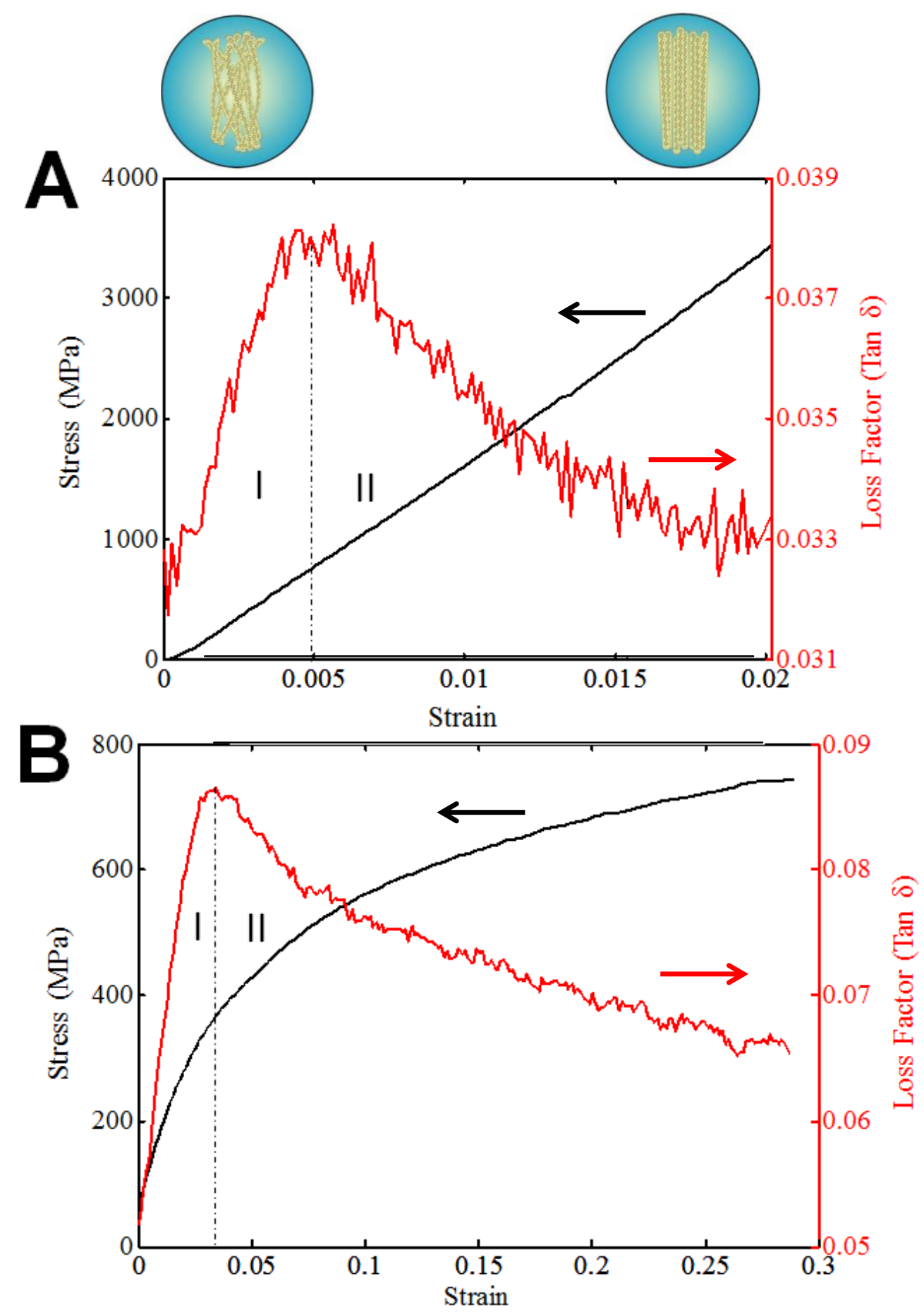

Figure 1. Tensile Engineering Stress-Strain Curves and Strain-Dependent Loss Factor Plots of Kevlar 49 and Nomex Fibers.

A. Plot of stress (black, left y-axis) and loss factor (red, right y-axis) versus strain in Kevlar 49, shown up to a failure strain of 0.02 . Microstructural changes are illustrated by cartoon images above the regimes. B. Plot of stress (black, left y-axis) and loss factor (red, right y-axis) versus strain in Nomex shown at strains up to failure. 

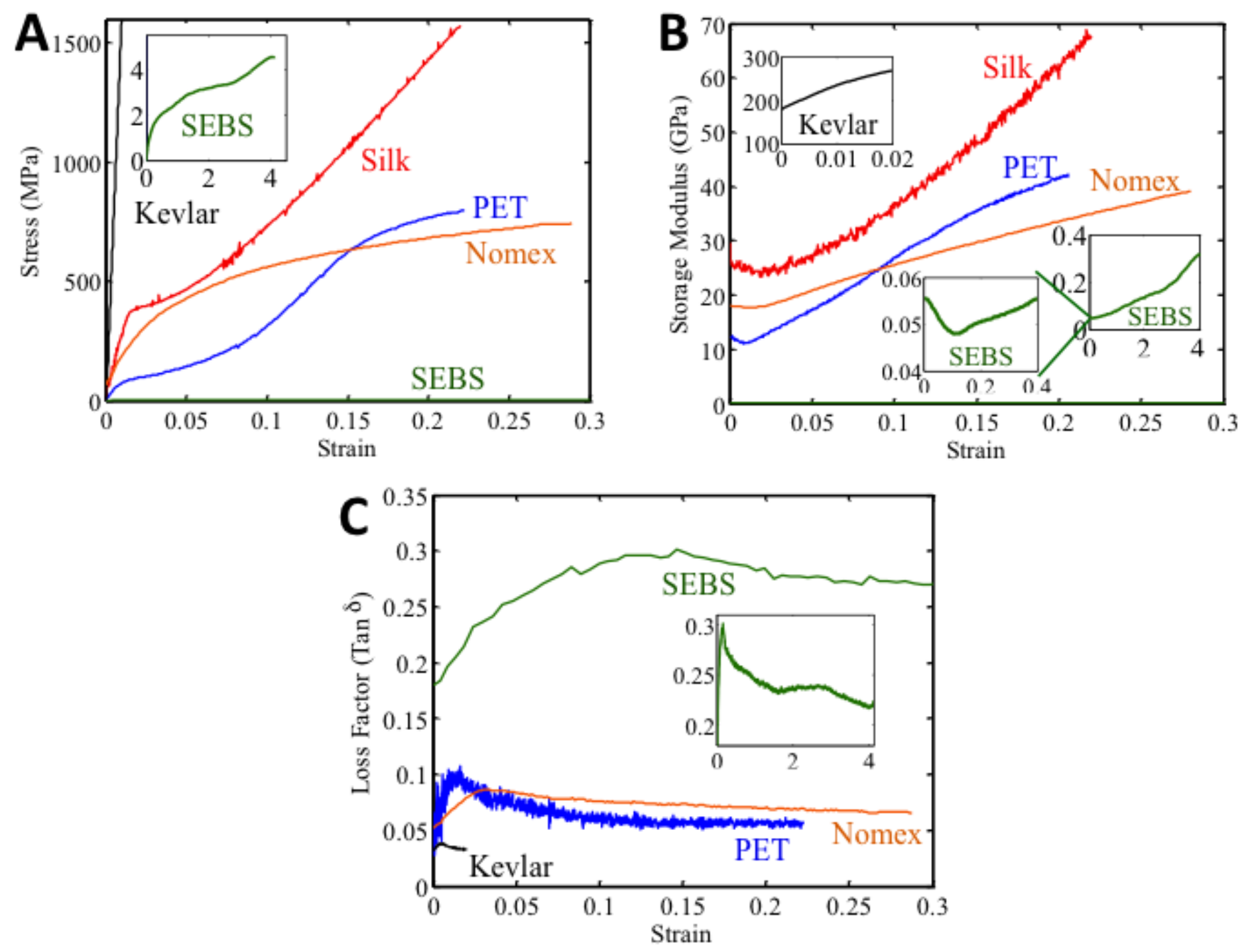

Figure 2. Comparison of the Quasistatic and Strain-Dependent Dynamic Mechanical Properties of Kevlar and Nomex as Compared to other Polymers.

A. Engineering stress-strain curves shown at strains up to failure for Kevlar (black), Nomex (orange), dragline spider silk (red), polyester (blue), and SEBS rubber (green).

B. Storage modulus as a function of strain at strains up to failure for Kevlar (black), Nomex (orange), dragline spider silk (red), polyester (blue), and SEBS rubber (green).

C. Loss factor versus strain to failure for Kevlar (black), Nomex (orange), polyester (blue), and SEBS rubber (green). See supplementary online information for data on loss factor as a function of strain to failure for dragline spider silk. 


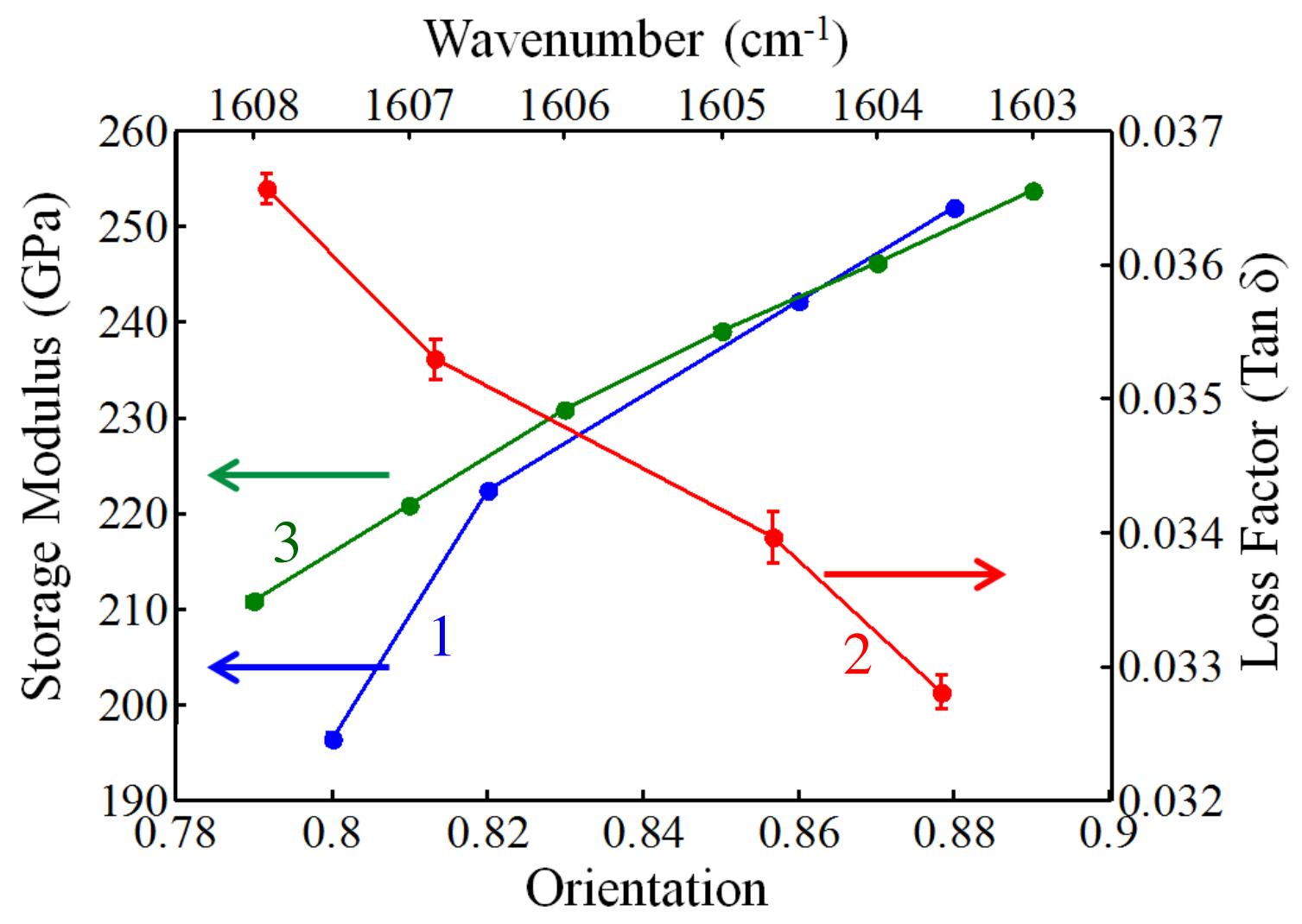

Figure 3. Variation in the Strain-Dependent Dynamic Mechanical Properties of Kevlar with Structural Changes. Plot correlating experimentally measured storage modulus and loss tangent for Kevlar 49 fibers with previous studies of other Kevlar fibers with differing structural fibril orientation. Curves 1 (blue) and 2 (red) correlate the storage modulus and loss factor, respectively, of Kevlar 49 fibers with fibril orientation. Curve 3 (green) correlates the storage modulus of Kevlar 49 fibers to the shift in Raman absorption frequency of the phenylene-pi bonds in Kevlar. Increasing fiber strain corresponds to a lower absorption wavenumber. 


\section{ASSOCIATED CONTENT}

Supporting Information. Materials and methods, the basic set of equations governing continuous dynamic analysis, and data on the dynamic mechanical properties of Kevlar and Nomex.

*e-mail: (R.O.R.) roritchie@lbl.gov

\section{AUTHOR INFORMATION}

\section{Corresponding Author}

\section{Author Contributions}

The manuscript was written through contributions of all authors. All authors have given approval to the final version of the manuscript. $¥$ These authors contributed equally.

\section{Notes}

The authors declare no competing financial interest. 


\section{Acknowledgments and Funding Sources}

Studies on Kevlar, SEBS, and Nomex fibers were supported by the Office of Science, Office

of Basic Energy Sciences, Division of Materials Sciences and Engineering, of the U.S. Department of Energy under contract DE-AC02-05CH11231, specifically through the Lawrence Berkeley National Laboratory (LBNL) Inorganic/Organic Nanocomposites NSET Program (for S.N.R., A.P.A.). The mechanical characterization was provided by LBNL's Mechanical Behavior of Materials Program, also supported by Office of Science, Office of Basic Energy Sciences, Division of Materials Sciences and Engineering, of the U.S. Department of Energy (for R.O.R). Additionally, L.L. acknowledges of NSF Grant ECCS-0901864 for mechanical characterization support. The authors thank Prof. Cheryl Hayashi and Dr. James Starrett for providing the spider silk.

\section{ABBREVIATIONS}

CDA, continuous dynamic analysis; DMA, dynamic mechanical analysis; PEB, poly(ethylenebutylene); PET, polyethylene terephthalate; PS, polystyrene; SAXS, small-angle x-ray scattering; SEBS, styrene- $b$-ethylene-butylene- $b$-styrene; WAXD, wide-angle x-ray diffraction. 


\section{REFERENCES}

1. Yang, H. H. Kevlar Aramid Fiber, 1st ed.; Wiley: Chichester, 1993; Vol. 1

2. Young, R. J.; Lu, D.; Day, R. J.; Knoff, W. F.; Davis, H. A. J. Mater. Sci., 1992, 27, 54315440 .

3. Kunugi, T; Watanabe, H.; Hashimoto, M. J. App. Polym. Sci., 2003, 24, 1039-1051.

4. Blackledge, T. A.; Swindeman, J. E.; Hayashi, C. Y. J. Exp. Biol., 2005, 208, 1937-1949

5. Ran, S.; Fang, D.; Zong, X.; Hsiao, B. S.; Chu, B.; Cunniff, P. M. Polymer, 2001, 1601-1612

6. Prasad, K.; Grubb, D. T. J. App. Polym. Sci., 1990, 41, 2189-2198

7. Kakida, H.; Chatani, Y.; Tadokoro, H. J. Polym. Sci., Polym. Phys. Ed., 2003, 14, 427-435

8. Menard, K.P. Dynamic Mechanical Analysis: A Practical Introduction, 2nd ed.; CRC Press: Boca Raton, 2008

9. Griffiths, J. R.; Salanitri, V. R. J. Mater. Sci. 1980, 15, 491-496

10. Raja, S. N.; Olson, A. C. K.; Thorkelsson, K.; Luong, A. J., Hsueh, L.; Chang, G.; Gludovatz, B; Lin, L.; Xu, T.; Ritchie, R. O.; Alivisatos, A. P. Nano Lett., 2013, 13, 39153922

11. Thorn, A. D.; Robinson, R. A. In Rubber Products and Manufacturing Technology, 1st ed.; Bhowmick, A. K.; Hall, M. M.; Benarey, H. A., Eds.; CRC Press: New York; Vol. 1

12. Downing, J. W.; Newell, J. A. J. App. Polym. Sci., 2004, 91, 417-424

13. Hashimoto, T.; Fujimura, M.; Saijo, K.; Kawai, H.; Diamant, J.; Shen, M. In Multiphase Polymers, 1st ed.; Cooper, S. L.; Estes, G. M., Eds.; American Chemical Society: Washington, DC, 1979; Vol. 176, p 257-275. 
Supplementary Information

\title{
Strain-Dependent Dynamic Mechanical Properties of Kevlar to Failure: Structural Correlations and Comparisons to other Polymers
}

\author{
Shilpa N. Raja ${ }^{1,2,}$ Sandip Basu ${ }^{3}$, Aditya M. Limaye ${ }^{4}$, Christina M. Hyland ${ }^{4}$, Turner \\ J. Anderson ${ }^{5}$, Liwei Lin $^{5}$, A. Paul Alivisatos ${ }^{1,2,6}$, Robert O. Ritchie $*^{1,2,5}$ \\ ${ }^{1}$ Materials Sciences Division, Lawrence Berkeley National Laboratory, Berkeley, CA 94720 \\ ${ }^{2}$ Department of Materials Science \& Engineering, University of California, Berkeley, CA 94720 \\ ${ }^{3}$ Agilent Technologies, 4330 W. Chandler Blvd, Chandler, AZ 85226 \\ ${ }^{4}$ Department of Chemical Engineering, University of California, Berkeley, CA 94720 \\ ${ }^{5}$ Department of Mechanical Engineering, University of California, Berkeley, CA 94720 \\ Department of Chemistry, University of California, Berkeley, CA 94720 \\ *Corresponding author email: roritchie@lbl.gov
}

\section{Materials and Methods}

\section{Materials}

Kevlar 49 fibers $(10-\mu \mathrm{m}$ diameter) were obtained from Dupont. Nomex fibers $(10-\mu \mathrm{m}$ diameter) were purchased from The Thread Exchange, 10- $\mu \mathrm{m}$ diameter). SEBS (MD 1537) was obtained from Kraton corporation; films of Kraton SEBS of thickness $\sim 100$ $\mu \mathrm{m}$ were obtained by casting from chloroform into a glass petri dish and drying under vacuum overnight. Strips of width 1-2 $\mathrm{mm}$ were cut for testing. Polyethylene terephthalate (PET) was obtained from Goodfellow Corporation. Black widow dragline spider silk ((Lactodecterus hesperus) was kindly provided by Prof. Cheryl Hayashi and Dr. James Starrett.

\section{Methods}

\section{Quasistatic and Continuous Dynamic Tensile Testing}

Quasistatic and continuous dynamic tensile testing was performed in a single test using an Agilent T-150 nano-mechanical tensile testing machine. For Kevlar, Nomex, and SEBS, the harmonic force amplitude was $4.5 \mathrm{mN}$, and the oscillation frequency for was $20 \mathrm{~Hz}$. For PET, the harmonic force amplitude was $2 \mathrm{mN}$, and the oscillation frequency was $20 \mathrm{~Hz}$. For Argiope spider silk, the harmonic force amplitude was $2 \mathrm{mN}$, and the oscillation frequency was $20 \mathrm{~Hz}$. The maximum harmonic displacement was $27 \mu \mathrm{m}$ in the 
case of $4.5 \mathrm{mN}$ application, and $12 \mu \mathrm{m}$ in the case of $2 \mathrm{mN}$ force application. Quasistatic strain rates $\left(\mathrm{sec}^{-1}\right)$ of $1 \times 10^{-04}$ (Kevlar, spider silk, and PET), $2 \times 10^{-03}$ (Nomex), and $1 \mathrm{x}$ $10^{-02}(\mathrm{SEBS})$ were used.

Films were gripped directly in the T150 testing machine tensile grips, while fibers were glued to tabs with diamond-shaped cutouts using cyanoacrylate glue with a foam kicker to accelerate drying. Kevlar was glued with PolyZap glue and cured for $24 \mathrm{hr}$ before testing.

To obtain a statistical average of the Young's modulus, five or six samples were tested and the results averaged for each material.

Lines of best fit to Kevlar and Nomex storage modulus curves were obtained using leastsquares linear regression analysis in MATLAB.

\section{Continuous Dynamic Analysis}

The viscoelastic properties of a fiber under tension are generally measured by dynamic mechanical analysis (DMA), which applies a harmonic force $\left(F_{0}\right)$ on a specimen. The dynamic storage and loss moduli can then be measured from the resulting harmonic displacement $\left(z_{0}\right)$ and the phase lag $(\delta)$. The storage modulus, $E^{\prime}$, and the loss modulus, $E^{\prime \prime}$, can be defined from these parameters in the dynamic mechanical analysis.

$$
\begin{aligned}
E^{\prime} & =\frac{L}{A}\left(\frac{F_{0}}{z_{0}} \cos \delta\right) \\
E^{\prime \prime} & =\frac{L}{A}\left(\frac{F_{0}}{z_{0}} \sin \delta\right)
\end{aligned}
$$

where, $L$ and $A$ are the instantaneous length and cross-sectional area of the fiber. The loss factor, or $\tan (\delta)$, can be written as:

$$
\tan (\delta)=\frac{E^{\prime \prime}}{E^{\prime}}
$$

Such a dynamic characterization method is particularly useful for polymeric materials, which exhibit a highly viscoelastic response to applied stress. As such DMA testing can only be performed at low harmonic displacements, one of the main aspects of the current work was to additionally utilize continuous dynamic analysis (CDA) which, by employing a small harmonic displacement during tensile deformation, can combine the advantages of DMA with those of the tensile test to quantify the extent of viscoelasticity as a continuous function of strain (1). Using the phase lag between stress and strain extracted from CDA, we are able to use the relationships introduced above to measure the storage and loss moduli as continuous functions of strain. The use of the nano-mechanical actuating transducer in the Agilent T150 testing facility provides the ability to make such measurements over a broad range of quasi-static strains (1).

The Herman's Orientation factor, $f$, is defined as: 


$$
f=\frac{3 \cos (\phi)-1}{2}
$$

where $\varphi$ indicates the angle between the crystal plane of interest and a particular reference axis. The function equals unity when the plane is parallel to the reference axis, and -0.5 when the plane is perpendicular to this axis. A value of zero indicates completely random orientation.

The structural correlations presented in Figure 1D were created by taking data from previous structural studies of Kevlar, and plotting it against parameters from our CDA on Kevlar. The error bars were calculated by averaging the dynamic mechanical properties in a small range of strains near those at which structural data was available.

\section{Reference}

1. Basu, S.; Hay, J. L.; Swindeman, J. E.; Oliver, W. C. MRS Comm, 2014, 4, 25-29. 
Table of Contents (ToC) Figure



